\title{
Editorial
}

\section{Applied Mathematics for Engineering Problems in Biomechanics and Robotics}

\author{
Carlos Llopis-Albert $\mathbb{D}$, ${ }^{1}$ Francisco Rubio $\mathbb{D}^{1},{ }^{1}$ Shouzhen Zeng $\mathbb{D}^{2},{ }^{2}$ and Huchang Liao $\mathbb{D}^{3}$ \\ ${ }^{1}$ Centro de Investigación en Ingeniería Mecánica (CIIM), Universitat Politècnica de València, Camino de Vera s/n, \\ 46022 Valencia, Spain \\ ${ }^{2}$ Ningbo University, Ningbo 315211, China \\ ${ }^{3}$ Sichuan University, Chengdu, Sichuan 610065, China \\ Correspondence should be addressed to Huchang Liao; liaohuchang@163.com
}

Received 5 February 2019; Accepted 6 February 2019; Published 21 April 2019

Copyright (C) 2019 Carlos Llopis-Albert et al. This is an open access article distributed under the Creative Commons Attribution License, which permits unrestricted use, distribution, and reproduction in any medium, provided the original work is properly cited.

This special issue brings together a collection of mathematical work relating to the disciplines of biomechanics and robotics, which are increasingly closely linked. On the one hand, biomechanics is the application of the principles and techniques of mechanics to the structure and functions of living organisms. On the other hand, robotics is the branch of science and technology that deals with the design, construction, operation, and application of robots and automated mechanical systems. They promise to be two of the most influential lines of research in the twenty-first century, since they generate innovation in a wide range of research fields. They encompass areas such as medicine, healthcare, sports, logistics and distribution, manufacturing industries, and business technology. Both disciplines are inherently multidisciplinary by nature, involving mathematics, physics, biology, and computing. The intention of this special issue is therefore to deal with mathematical work related to medical imaging and visualization, bioinformatics, exoskeletons, microelectromechanical systems and nanotechnology, new biomaterials and sensors, medical robotics, healthcare robots, injury prevention and rehabilitation, enhancement of patient and worker safety and quality, ergonomics, production, logistics and distribution applications, socioeconomic and management issues, and decision-making processes related to these disciplines.

This special issue is devoted to applied mathematics in both biomechanics and robotics and to their integration. Consequently, the objective is to disseminate advanced mathematical research on biomechanics and robotics and their applications while promoting integration between these disciplines. The published articles address significant issues and contribute to the development of new concepts, methodologies, applications, trends, and knowledge in science. In this regard, the topics covered by this special issue include the following: biomechanics and robotics: use of techniques from computational mechanics, such as Finite Element Analysis (FEA) and Multibody Dynamics Analysis (MDA); new mathematical approaches, innovations, and challenges in biomechanics and robotics; biomechanics and robotics: new trends in modeling and simulation; kinematics, dynamics, and optimization procedures in biomechanics and robotics; computer-based methods in biomechanics, biomedical engineering, and robotics; applied mathematics to deal with manufacturing, production, logistics and distribution, product design, ergonomics, management and socioeconomic aspects, and (fuzzy) decision-making processes related to biomechanics and robotics; topics related to mathematical approaches to human motion analysis, virtual prototypes, diagnosis, injury prevention, treatment, and rehabilitation; sports performance, training techniques, and development of materials and equipment by means of biomechanics and robotics; biomechanics and robotics applications in medicine, work efficiency, and risk factors among assembly line workers.

For this special issue, we requested high-quality original research articles in the above-mentioned topics. The number 
of accepted submissions in the special issue is 16 , which have been published after a fair and rigorous review process organized by the guest editorial team. The accepted papers cover a wide range of topics and a concise description of them follows.

In the paper entitled "Existence and Stability of Periodic Solution Related to Valveless Pumping” by B. Dorociaková et al., an application in nanotechnology is presented using a valveless pumping approach, also known as the Liebau effect. It was described as the unidirectional flow of liquid in a system without valves that is caused by the asymmetrical placement of the periodically working pump.

H. E. Jaramillo set out and discussed the importance of the intervertebral disc in the mechanical behavior of the human spine using a finite element model of the human spine in the paper "Evaluation of the Use of the Yeoh and MooneyRivlin Functions as Strain Energy Density Functions for the Ground Substance Material of the Annulus Fibrosus".

The paper entitled "A Reliability-Based Consensus Model for Multiattribute Group Decision-Making with Analytically Evidential Reasoning Approach" by Y.-W. Du et al. presents a study that provides a reliability-based consensus model for multiattribute group decision making with an analytically evidential reasoning approach.

B. Liu et al. developed a real-time evacuation route approach based on emotion and geodesics under the influence of individual emotion and multihazard circumstances in the paper entitled "An Evacuation Route Model of Crowd Based on Emotion and Geodesic".

Dealing with position tracking control in the paper "Position Tracking Control of PMSM Based on Fuzzy PIDVariable Structure Adaptive Control", P. Pei et al. propose a novel fuzzy PID-variable structure adaptive control for position tracking of a permanent magnet synchronous motor, which is used in an electric extremity exoskeleton robot.

In the paper entitled "Human-Robot Interaction and Demonstration Learning Mode Based on Electromyogram Signal and Variable Impedance Control", R. Wu et al. simulate the properties of a variable admittance controller and a variable impedance controller, showing the good performance of these two controllers under trajectory tracking and physical interaction. In addition, a new mode of learning from demonstration that conforms to human intuition was developed by combining the electromyogram (EMG) signals and variable impedance (admittance) controller in a dragging demonstration.

Y.-W. Du et al. introduce a "Mass CollaborationDriven Method for Recommending Product Ideas Based on Dempster-Shafer Theory of Evidence".

In the paper entitled "Decision-Maker's Risk Preference Based Intuitionistic Fuzzy Multiattribute Decision-Making and Its Application in Robot Enterprises Investment", L. Zhou and Q. Wang developed an intuitionistic fuzzy dependent hybrid weighted operator and proposed an intuitionistic fuzzy multiattribute decision-making method, which was verified in a robot manufacturing investment case study.

In the paper entitled "Extension Dependent Degree Method with Mapping Transformation for Three-Parameter Interval Number Decision Making”, L.-B. Xu et al. put forward a new decision-making approach and framework based on extension simple dependent degree. It makes it possible not only to perform uncertain analysis of decision results by different settings of attitude coefficients, but also to take dynamic analysis and rule finding by some extension of the interval mapping transformation method.

In the paper entitled "Bifurcation Analysis of Stick-Slip Motion of the Vibration-Driven System with Dry Friction" regarding the problem of vibration-driven systems, which can move due to the periodic motion of the internal mass and the dry friction, P. Li and Z. Jiang proposed to model their system as a Filippov system with the property of stick-slip motion.

In the paper entitled "Nonlinear Vibration of an Elastic Soft String: Large Amplitude and Large Curvature", D.-M. Zhao et al. addressed the nonlinear vibration of an elastic string with a large amplitude and large curvature, which was systematically investigated using the Hamilton principle, the modified complex normal form method, and the finite difference scheme.

L. Yu et al. dealt with multicriteria decision analysis methods by presenting a new assessment approach based on the analysis of Technique for Order of Preference by Similarity to Ideal Solution (TOPSIS) evaluation methods in the paper entitled "A Study on the Application of Coordinated TOPSIS in Evaluation of Robotics Academic Journals".

In the paper entitled "Workspace Analysis of a Hybrid Kinematic Machine Tool with High Rotational Applications", H. Zhang et al. proposed a novel parallel manipulator with one translational and two rotational degrees of freedom that can be used to form a five-degree-of-freedom hybrid kinematic machine tool for large heterogeneous complex structural component machining in aerospace field.

The paper entitled "Extended Version of Linguistic Picture Fuzzy TOPSIS Method and Its Applications in Enterprise Resource Planning Systems" by S. Zeng et al. uses uncertain information in terms of linguistic picture fuzzy numbers. The decision-maker provides membership, neutral, and nonmembership fuzzy linguistic terms to represent uncertain assessments information of alternatives in linguistic multicriteria decision making, in which a new hamming distance measure between two linguistic picture fuzzy numbers is defined in order to introduce the extended version of TOPSIS method.

J. Bai et al. in the paper entitled "EO Sensor Planning for UAV Engineering Reconnaissance Based on NIIRS and GIQE" developed a method to solve the problem of poor image quality caused by the flight altitude not meeting the mission requirements based on National Imagery Interpretability Rating Scale (NIIRS), the general image quality equation (GIQE), and the geometrical property of the ground sampled distance (GSD).

In the paper entitled "Modal Decoupled Dynamics FeedForward Active Force Control of Spatial Multi-DOF Parallel Robotic Manipulator", X. Niu et al. proposed an intelligent robotic spine brace for the treatment of scoliosis. In order to solve the disadvantages of the parallel mechanism, they present a novel active force control structure, modal space dynamic feed-forward (MSDF) force control strategy. 


\section{Conflicts of Interest}

The guest editors declare that there are no conflicts of interest regarding the publication of this special issue.

\section{Acknowledgments}

We would like to thank all the authors for their excellent contributions and the reviewers for their valuable help; their feedback was very useful in improving the quality of the papers submitted.

Carlos Llopis-Albert Francisco Rubio Shouzhen Zeng Huchang Liao 


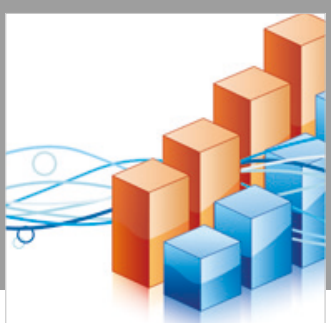

Advances in

Operations Research

\section{-n-m}
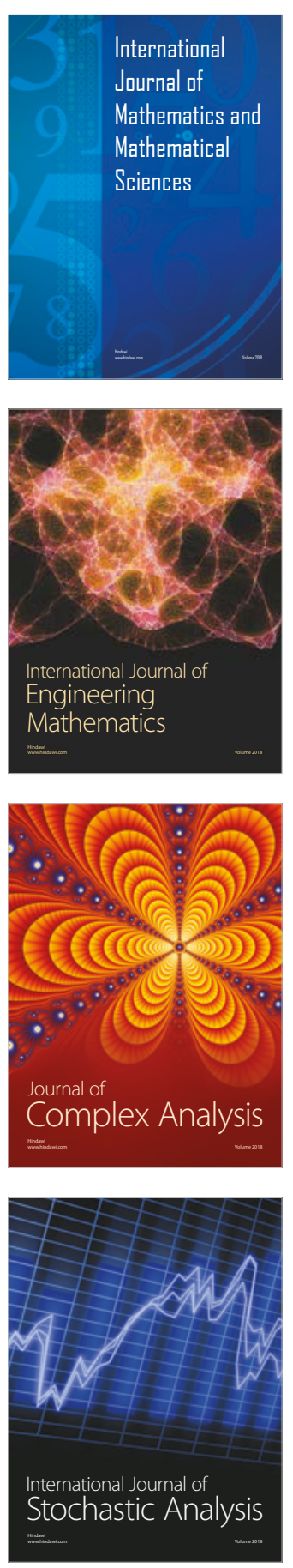
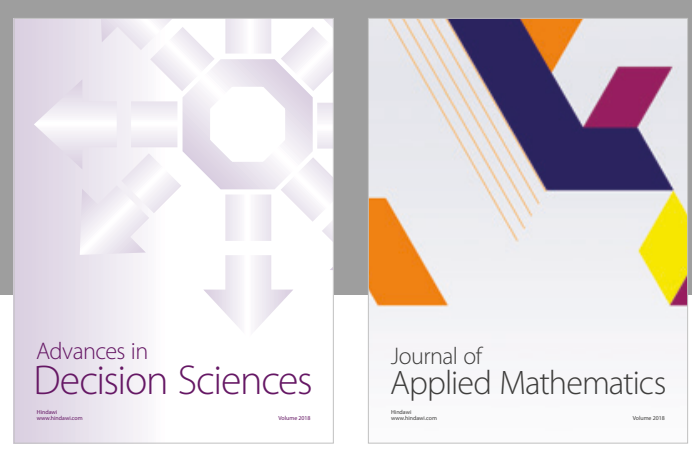

Journal of

Applied Mathematics
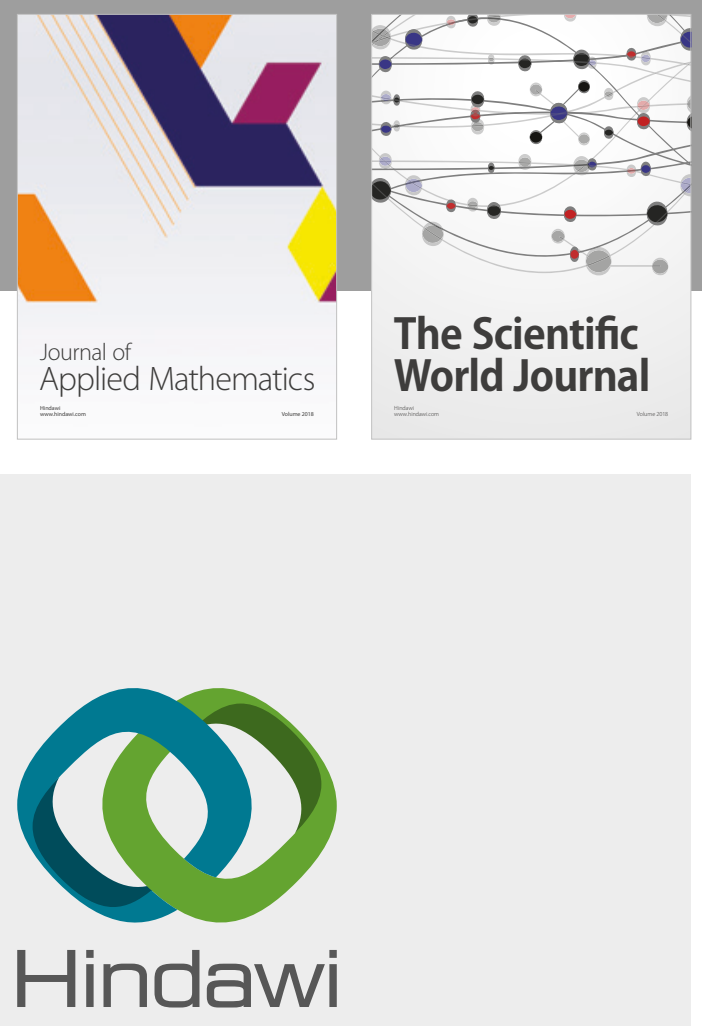

Submit your manuscripts at

www.hindawi.com

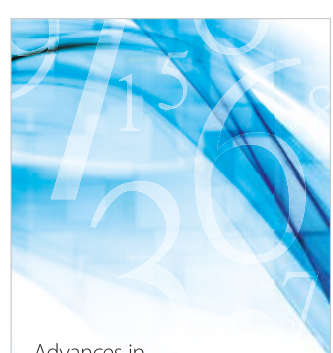

Advances in
Numerical Analysis
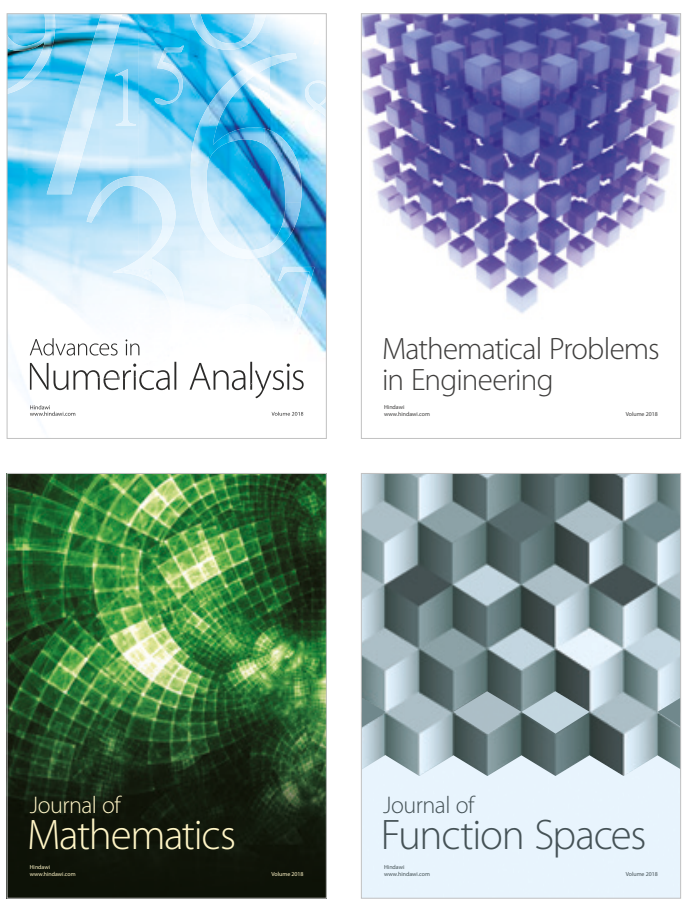

Mathematical Problems in Engineering

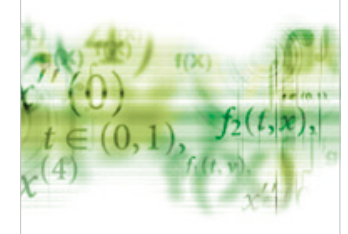

International Journal of

Differential Equations

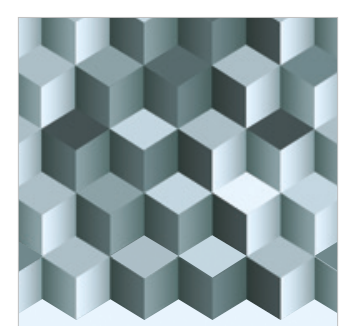

Journal of

Function Spaces

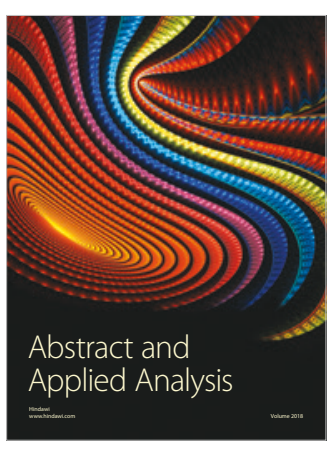

The Scientific

World Journal

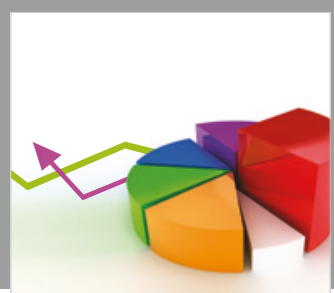

Journal of

Probability and Statistics
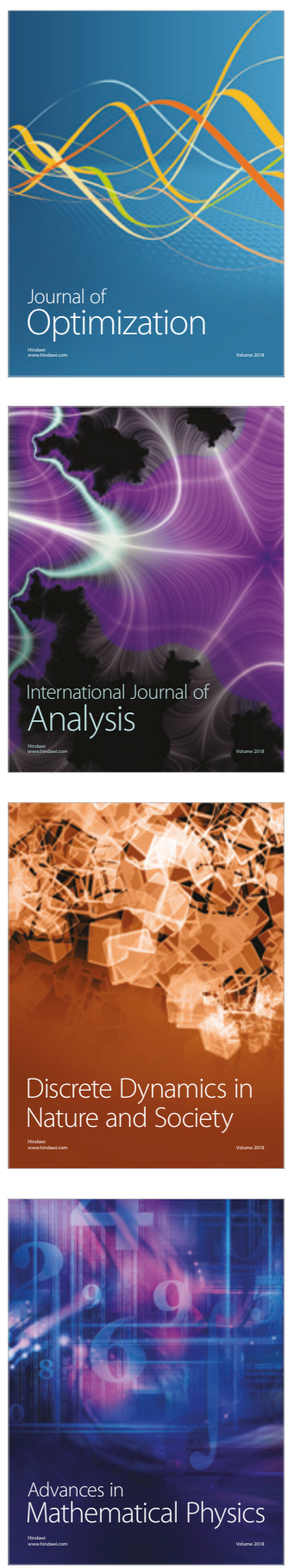